\title{
Gene expression profiling of epithelial ovarian cancer reveals key genes and pathways associated with chemotherapy resistance
}

\author{
M. Zhang and S.C. Luo \\ Oncology Department, \\ Sichuan Provincial Hospital and Sichuan Academy of Medical Science, \\ Chengdu, Sichuan, China \\ Corresponding author: S.C. Luo \\ E-mail: shucunluosd@163.com \\ Genet. Mol. Res. 15 (1): gmr.15017496 \\ Received August 21, 2015 \\ Accepted November 6, 2015 \\ Published January 22, 2016 \\ DOI http://dx.doi.org/10.4238/gmr.15017496
}

ABSTRACT. The aim of this study is to analyze gene expression data to
identify key genes and pathways associated with resistance to platinum-
based chemotherapy in epithelial ovarian cancer (EOC) and to improve
clinical treatment strategies. The gene expression data set was downloaded
from Gene Expression Omnibus and included 12 chemotherapy-resistant
EOC samples and 16 chemotherapy-sensitive EOC samples. A differential
analysis was performed to screen out differentially expressed genes
(DEGs). A functional enrichment analysis was conducted for the DEGs
using the database for annotation, visualization, and integration discovery.
A protein-protein interaction (PPI) network was constructed with information
from the human protein reference database. Pathway-pathway interactions
were determined with a test based on the hypergeometric distribution.
A total of 1564 DEGs were identified in chemotherapy-sensitive EOC,
including 654 upregulated genes and 910 downregulated genes. The top
three upregulated genes were HIST1H3G, AKT3, and RTN3, while the top 
three downregulated genes were NBLA00301, TRIM62, and EPHA5. A Gene Ontology enrichment analysis showed that cell adhesion, biological adhesion, and intracellular signaling cascades were significantly enriched in the DEGs. A KEGG pathway enrichment analysis revealed that the calcium, mitogen-activated protein kinase, and $B$ cell receptor signaling pathways were significantly over-represented in the DEGs. A PPI network containing 101 interactions was acquired. The top three hub genes were RAC1, CAV1, and BCL2. Five modules were identified from the PPI network. Taken together, these findings could advance the understanding of the molecular mechanisms underlying intrinsic chemotherapy resistance in EOC.

Key words: Epithelial ovarian cancer; Chemotherapy resistance; Differentially expressed genes; Functional enrichment analysis; Protein-protein interaction network; Module

\section{INTRODUCTION}

Ovarian cancer is the seventh most common cancer and the eighth most common cause of death from cancer in women; it usually has a relatively poor prognosis (Stewart and Wild, 2014). Treatment includes chemotherapy, surgery, and sometimes radiotherapy. Chemotherapy resistance is a major obstacle in treating patients with ovarian cancer (Gatti and Zunino, 2005). Based on the progression-free survival (PFS) after chemotherapy, patients are classified as chemotherapy-sensitive (PFS > 18 months) or chemotherapy-resistant (PFS < 6 months). Resistance to platinum-based chemotherapy is multifactorial and is either intrinsic or acquired with drug exposure. It has been said that rapid relapses of platinum-resistant diseases are due to intrinsically resistant cancer cells (Cooke and Brenton, 2011). An active interaction between the drug and tumor microenvironment may also contribute to the gain of resistance to chemotherapy via dysregulation of various pathways (Bachvarov et al., 2006). Therefore, the identification of biomarkers as well as critical pathways associated with chemotherapy resistance could enable targeted therapy at an earlier time point in the disease process.

Many studies have investigated the relationships between gene expression profiles in ovarian cancer and resistance to chemotherapy (Jazaeri et al., 2005; L'Espérance et al., 2006; Osterberg et al., 2009; Helleman et al., 2010; Konstantinopoulos et al., 2010; Koti et al., 2013). L'Espérance et al. (2006) first suggested that intrinsic and acquired chemotherapy resistance may be attributed to the combined action of different factors implicated in mechanisms of chemoresistance, tumor invasion and progression, and control of cell proliferation. Using high-resolution whole genome array comparative genomic hybridization, Osterberg et al. (2009) identified several specific genetic alterations associated with chemotherapy resistance in advanced ovarian serous carcinomas. Furthermore, Helleman et al. (2010) implicated the involvement of oxidative stress response mediated by Nrf2, TP53 signaling, and transforming growth factor (TGF)- $\beta$ signaling in platinumbased chemotherapy resistance. In addition, Koti et al. (2013) found that insulin-like growth factor 1/phosphoinositide 3-kinase (PI3K)/nuclear factor kappa B /extracellular signal-regulated kinase gene signaling networks are associated with chemotherapy resistance in epithelial ovarian cancer (EOC). Nevertheless, the currently available knowledge is not yet sufficient to improve clinical 
outcomes associated with chemotherapy resistance of EOC.

In the present study, we analyzed gene expression data with various bioinformatic tools to obtain more information about chemotherapy resistance of EOC. We performed differential analyses and protein-protein interaction (PPI) analyses to identify the key genes. We carried out pathwaypathway interaction analyses to uncover the relevant pathways contributing to chemotherapy resistance. Overall, these findings could advance the understanding of the molecular mechanisms underlying intrinsic chemotherapy resistance in EOC.

\section{MATERIAL AND METHODS}

\section{Gene expression data}

The gene expression data set (accession No. GSE51373) (Koti et al., 2013) was downloaded from the Gene Expression Omnibus database, which included 28 EOC patients who were divided into two groups based on their varying sensitivities to platinum-based chemotherapy by using PFS as an indicator. Twelve EOC patients were regarded as chemotherapy-resistant with PFS less than 6 months and 16 EOC patients were considered as chemotherapy-sensitive with PFS greater than 18 months. Gene expression levels were measured using the Human Genome U133 Plus 2.0 Array (Affymetrix, Santa Clara, CA, USA). Probe annotations were also acquired.

\section{Pre-treatment and differential analyses}

Raw data in the CEL format were pre-treated with the R package affy (Irizarry et al., 2003). Background correction and MAS5.0 normalization were then performed. Probes without gene annotations and those mapping to more than one gene were removed from the list.

Differential analysis was performed between the platinum-resistant group and the platinumsensitive group using the R package limma (Smyth, 2004). The cut-off values were set to llog(Fold Change) $\mid>1.5$ and $P$ value $<0.05$ to screen out the differentially expressed genes (DEGs).

\section{Functional enrichment analysis}

A functional enrichment analysis was conducted using the database for annotation, visualization and integration discovery (DAVID) (https://david.ncifcrf.gov/) (Huang et al., 2007). The EASE (Expression Analysis Systematic Explorer) test method was used in the analysis and an EASE value of 0.05 was set as the threshold.

\section{Construction of PPI network}

APPI network is a useful tool to identify critical genes and molecular mechanisms underlying platinum-based chemotherapy resistance. A PPI network was constructed for the DEGs using information from the human protein reference database (HPRD) (Keshava Prasad et al., 2009) and visualized with Cytoscape (Kohl et al., 2011). The proteins in the network serve as the nodes, and each pairwise protein interaction is represented by an undirected link and the degree of a node corresponds to the number of interactions of a protein. Degrees of interaction were calculated for each node. Hub genes were then removed according to the degree. In addition, modules were 
screened with the clique percolation method of the CFinder software (Adamcsek et al., 2006).

\section{Construction of pathway-pathway interaction network}

To further analyze the distribution pattern of resistance-related DEGs, a pathway-pathway interaction network was constructed. The pathway-pathway interaction was defined as follows: if DEG $a$ in pathway $A$ interacted with DEG $b$ in pathway $B$, then pathway $A$ is said to interact with pathway $B$. The PPI network was constructed with information from the HPRD and the following treatments were applied: 1) self-interaction proteins were removed and the remaining proteins were mapped to Entrez Gene; 2) these genes were compared with the genes identified in the two groups of microarray data and a total of 31,656 overlapping genes were obtained with the information from the PPI.

A cumulative hypergeometric probability distribution model was used to evaluate the significance of interaction between two pathways. The $\mathrm{P}$ value was calculated according to Equation 1, and 0.05 was set as the cut-off to screen out significant pathway-pathway interactions. Overlapping genes between two pathways were removed before calculation.

$$
p=1-\sum_{k=0}^{m-1} \frac{\left(\begin{array}{c}
M \\
k
\end{array}\right)\left(\begin{array}{c}
N-M \\
n-k
\end{array}\right)}{\left(\begin{array}{c}
N \\
n
\end{array}\right)}
$$

where $N$ is the number of PPIs involving all the DEGs; $M$ is the number of PPIs involving genes from pathway $A$ (the interaction contains at least one protein product of DEGs); $n$ is the number of PPIs involving genes from pathway $B$ (the interaction contains at least one protein product of DEGs); and $k$ is the number of PPIs between pathway $A$ and pathway $B$ (the interaction contains at least one protein product of DEGs).

\section{RESULTS}

\section{DEGs}

A total of 1564 DEGs were identified to be relevant in chemotherapy-sensitive EOC, of which 654 genes were upregulated and 910 genes were downregulated. DEGs were then sorted according to the $\mathrm{P}$ value. The top three upregulated genes were HIST1H3G, v-akt murine thymoma viral oncogene homolog 3 (AKT3), and reticulon 3 (RTN3). The top three downregulated genes were NBLA00301, tripartite motif containing 62, and Eph receptor A5 (EPHA5). Box plots were generated to visualize the differences in gene expression levels between the chemotherapysensitive group and the chemotherapy-resistant group (Figure 1).

\section{Functional enrichment analysis result}

A Gene Ontology (GO) enrichment analysis and a KEGG pathway enrichment analysis were performed for the 1564 DEGs. A P value of 0.05 was set as the threshold to screen out 
significant terms. The results are shown in Tables 1 and 2. Cell adhesion, biological adhesion, and intracellular signaling cascades were significantly enriched in the DEGs. KEGG pathways such as the calcium, mitogen-activated protein kinase (MAPK), and B cell receptor signaling pathways were significantly over-represented in the DEGs.
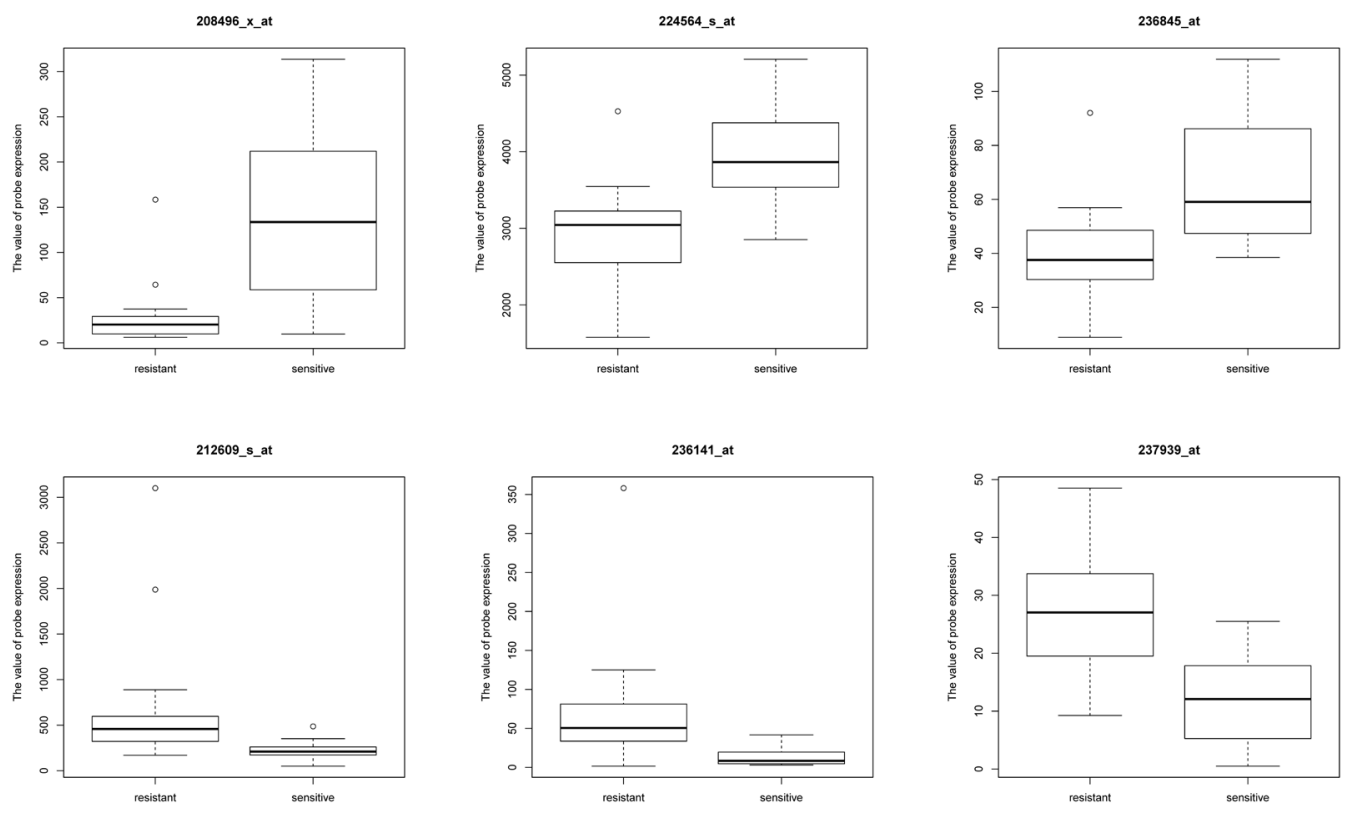

Figure 1. Box plots of expression levels of the top three upregulated genes (upper panels) and the top three downregulated genes (lower panels) in platinum-sensitive ovarian cancer.

\section{PPI network and modules}

A total of 268 DEGs were obtained from the top five GO terms and top five KEGG pathways, of which 91 were upregulated and 177 were downregulated. APPI network was constructed for the 268 DEGs with information from the HPRD. A total of $101 \mathrm{PPI}$ interactions were revealed and then visualized with Cytoscape (Figure 2).

The top three hub genes in the PPI network were ras-related $\mathrm{C} 3$ botulinum toxin substrate 1, caveolin 1, and B-cell CLL/lymphoma 2 (with degrees 9, 8 and 6). These genes were downregulated in platinum-sensitive serous ovarian cancer, which might play roles in the development of chemotherapy resistance.

Modules were screened from the whole network with the clique percolation method of CFinder. Five modules were obtained with $\mathrm{k}=3$, which might contribute to the gain of chemotherapy resistance in ovarian cancer (Figure 3).

\section{Pathway-pathway interaction network}

A pathway-pathway interaction network was constructed, which include 21 altered pathways that might underlie chemotherapy resistance in EOC (Figure 4). 
Table1.Top five GO terms significantly enriched in the differentially expressed genes.

\begin{tabular}{lcl}
\hline Term & Count & Genes \\
\hline $\begin{array}{l}\text { G0:0007155 } \\
\text { cell adhesion }\end{array}$ & \\
& LYVE1, COL9A1, LAMA1, LSAMP, CNTN3, VWF, PCDH18, \\
& COMP, CLDN5, CD36, MGP, RHOB, DCHS1, DSCAM, \\
& COL4A6, DSC2, F5, DDR2, COL2A1, FEZ1, CNTN5, \\
& PARVB, PTPRF, CLEC4M, PPFIA2, CD22, CD84, NELL1, \\
& DCBLD2, CDH19, ANGPTL3, IGFBP7, NRP2, CTGF, \\
& CD2AP, MPZ3, RAC1, NCAM1, NEGR1, TNXB, RS1, \\
& LAMA2, ECM2, PDPN, CNTNAP3, COL14A1, EMCN, \\
& NFASC, BCL2L11, FCGBP, KAL1, BOC, SSX2IP, BCL2, \\
& FNDC3A, SIRPA, MFAP4, PCDH1, SDK2, ARHGAP6, \\
& PCDH7, NRXN2, GNE, ADAM22, ADAM9, SPON2, SGCE, \\
& ADAM23, NID1, PCDHA2, BTBD9, MMRN1, SEMA5A, \\
& TROAP, PVR, CD209, FLRT2
\end{tabular}

G0:0022610 77

biological adhesion

GO:0007242

intracellular signaling

cascade

GO:0007610

rbehavior

GO:0007517

rmuscle organ

development
116

(n)

29 RAP, PVR, CD209, FLRT2

7.31E-06

LYVE1, COL9A1, LAMA1, LSAMP, CNTN3, VWF, PCDH18, COMP, CLDN5, CD36, MGP, RHOB, DCHS1, DSCAM, COL4A6, DSC2, F5, DDR2, COL2A1, FEZ1, CNTN5, PARVB, PTPRF, CLEC4M, PPFIA2, CD22, CD84, NELL1, DCBLD2, CDH19, ANGPTL3, IGFBP7, NRP2, CTGF, CD2AP, MPZL3, RAC1, NCAM1, NEGR1, TNXB, RS1, LAMA2, ECM2, PDPN, CNTNAP3, COL14A1, EMCN, NFASC, BCL2L11, FCGBP, KAL1, BOC, SSX2IP, BCL2, FNDC3A, SIRPA, MFAP4, PCDH1, SDK2, ARHGAP6, PCDH7, NRXN2, GNE, ADAM22, ADAM9, SPON2, SGCE, ADAM23, NID1, PCDHA2, BTBD9, MMRN1, SEMA5A, TROAP, PVR, CD209, FLRT2

INADL, F2, ZFP36, RACGAP1, STAT1, PTGER3, NDC80,

9.83E-05 IGF1, ESR1, PML, CD36, SQSTM1, FCER1A, CNIH3, RCAN1, DCLK2, PTHLH, ARHGEF7, CLEC4M, JAK3, NRAS, VAV2, TIAM1, CARD14, CTGF, RASL11A, DRD3, NMUR2, ZWINT, PRLR, PTPLAD1, IQGAP3, GNA12, KRAS, HINFP, SOCS1, DUSP1, GNG11, HIPK2, FHL2, ADCY2, ASB16, ECEL1, PGR, ARHGAP6, APBB2, CHEK1, RALBP1, H2AFX, IFT27, ADAM9, GNAL, PLD1, PRKAR1B, SP100, SIK1, KSR1, ARHGEF28, RABL6, SFN, PLCL1, GRM1, MKNK1, RHOB, FGF2, RHOJ, PRKD1, BUB1B, PKN1, RAB43, MYO9B, MAPK10, ITSN1, NR3C1, PRKD3, ARHGEF12, MAP2K7, TP63, KIT, MAP2K5, TRIM23, CD81, RASA1, DCBLD2, GEM, PRKCZ, ARF3, FGF13, INSR, HIST1H4A, RAC1, SOS1, MCF2, MAP4K2, TLR10, CBLB, CDC42BPG, HTR6, RASD1, HTR2A, PIK3C2A, NPR3, IL6ST, NDUFS4, TOP2A, GHR, CAV1, RASL11B, CIT, RASA3, PPAP2A, GAB1, BCL10, GRK5, BRIP1, CD209

CLN8, PLCL1, AFF2, COMT, GRM1, ALS2, P2RX3, GRIK2, FGF2, DSCAM, SNAP25, CCL28, ECSCR, GRIN1, SPTBN4, ATXN1, CXCL14, KIT, SLC1A2, TCF15, NRAS, PRKCZ, SEMA3F, FOXP2, NRP2, CCL21, DRD3, EPS8, RAC1, NMUR2, CCRL1, EGR2, EGR1, MAOA, TAS2R1, FOSB, KRAS, HTR2A, HIPK2, LTB4R2, KAL1, C5AR2, BCL2, PEX13, RALBP1, PLD1, PRKAR1B, USP46, STRBP, SLC18A2, FOS, CMTM7, NTRK2

FOXL2, ELN, CACNB2, AFG3L2, IGF1, PAX7, FOXP1,

2.63E-04 CACNG2, PLN, LAMA2,ALS2, FHL1, ATG5, RCAN1, DMD, PROX1, TP63, SMTN, CAV1, EGR3, CACNA1H, FKBP1A, SGCE, TCF15, FOXP2, SOX6, UNC45B, MYH11, ZFPM2 
Table2. Top five KEGG pathways significantly enriched in the differentially expressed genes.

\begin{tabular}{|c|c|c|c|}
\hline Term & Count & Genes & $P$ value \\
\hline $\begin{array}{l}\text { hsa04020: Calcium } \\
\text { signaling pathway }\end{array}$ & 25 & $\begin{array}{l}\text { SLC25A31, ITPR1, PTGER3, HTR6, RYR2 } \\
\text { GRM1, RYR3, TACR3, PLN, P2RX3, HTR2A, PPID, MYLK, } \\
\text { ADCY2, LTB4R2, PDE1C, GRIN1, PDGFRA, NOS1, SLC25A6, } \\
\text { GNAL, CACNA1H, ATP2B3, ATP2B4, MYLK3 }\end{array}$ & $3.68 \mathrm{E}-04$ \\
\hline $\begin{array}{l}\text { hsa04010: MAPK } \\
\text { signaling pathway }\end{array}$ & 31 & $\begin{array}{l}\text { CACNB2, MAP4K2, GNA12, KRAS, FGF7, CACNG2, PPP5C, } \\
\text { DUSP1, PTPRR, MKNK1, FGF2, PTPN5, HSPA1L, MAPK10, } \\
\text { PLA2G2A, FGF22, PDGFRA, MAP2K7, } \\
\text { MAP2K5, RASA1, CACNA1H, ARRB1, CDC25B, AKT3, } \\
\text { NRAS, NR4A1, FGF13, FOS, NTRK2, RAC1, SOS1 }\end{array}$ & 0.001962 \\
\hline hsa05218: Melanoma & 12 & $\begin{array}{l}\text { PDGFD, PIK3R3, NRAS,AKT3, FGF22, PDGFRA, } \\
\text { FGF2, FGF13, PDGFC, KRAS, FGF7, IGF1 }\end{array}$ & 0.005536 \\
\hline $\begin{array}{l}\text { hsa04662: B cell receptor } \\
\text { signaling pathway }\end{array}$ & 12 & $\begin{array}{l}\text { PIK3R3, NRAS, AKT3, VAV2, BCL10, CD22, KRAS, FOS, } \\
\text { RAC1, SOS1, CD81, CD79B }\end{array}$ & 0.008401 \\
\hline $\begin{array}{l}\text { hsa04664: Fc epsilon RI } \\
\text { signaling pathway }\end{array}$ & 12 & $\begin{array}{l}\text { MAPK10, PIK3R3, NRAS, AKT3, VAV2, PLA2G2A, } \\
\text { MAP2K7, MS4A2, KRAS, FCER1A, RAC1, SOS1 }\end{array}$ & 0.011218 \\
\hline
\end{tabular}

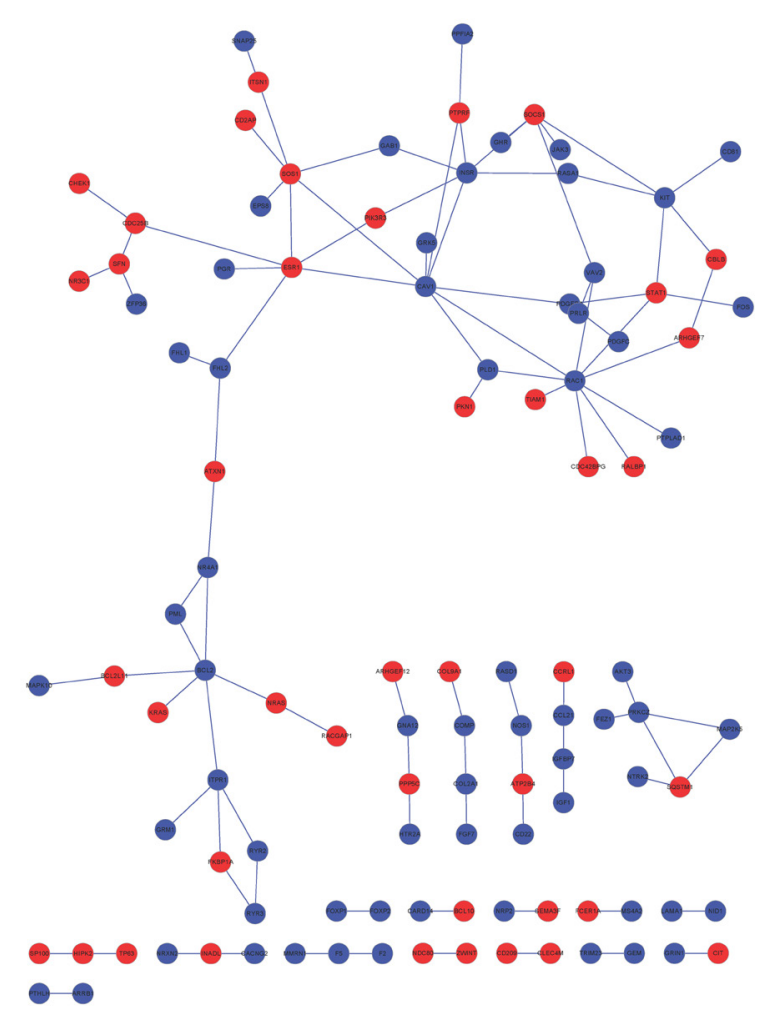

Figure 2. Protein-protein interaction network of the differentially expressed genes. Red circles represent upregulated genes while blue circles represent downregulated genes. 

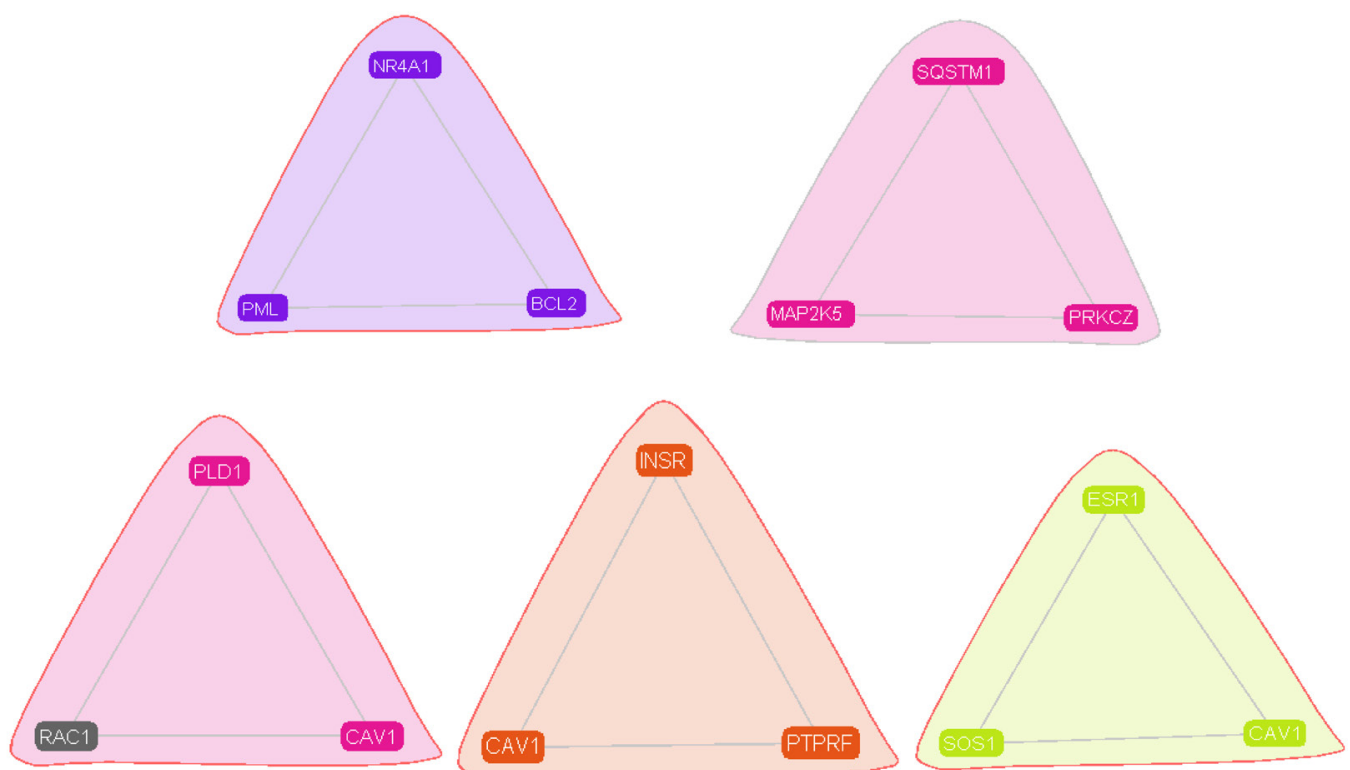

Figure 3. Modules identified from the protein-protein interaction network.
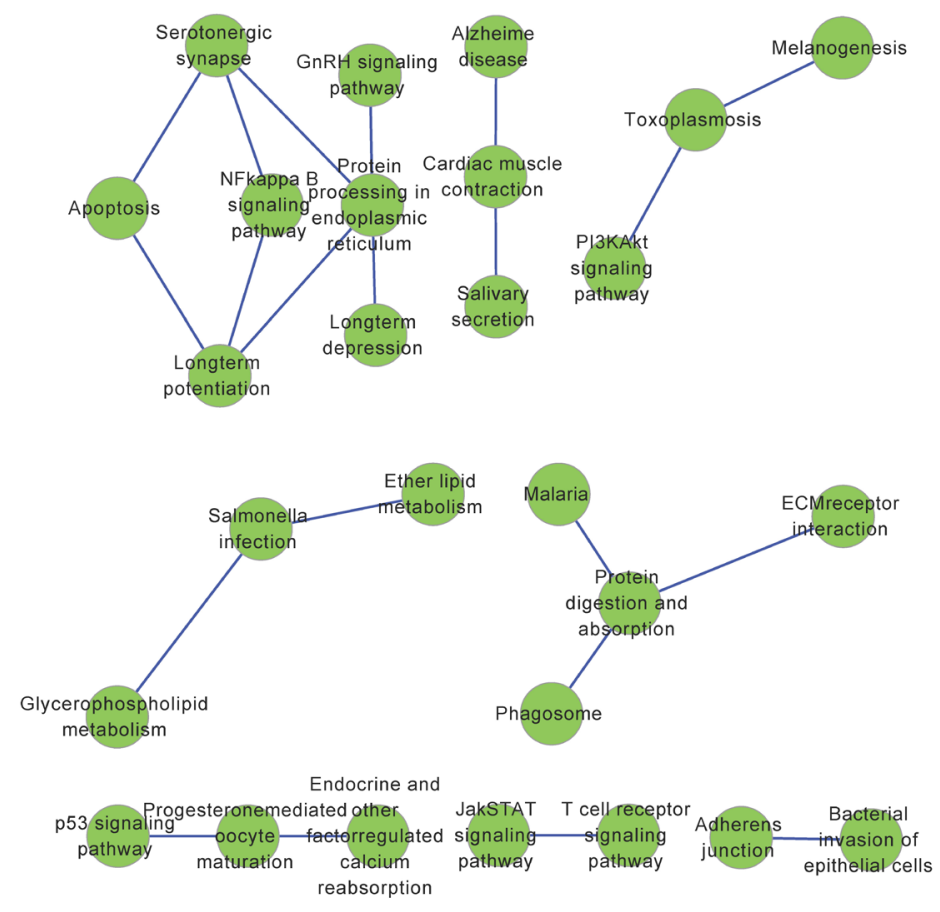

Figure 4. Pathway-pathway interaction network. 


\section{DISCUSSION}

In the present study, a comparative analysis of gene expression profiles was performed between chemotherapy-sensitive EOC and chemotherapy-resistant EOC. A total of 1564 DEGs were identified in chemotherapy-sensitive EOC, including 654 upregulated genes and 910 downregulated genes. The PPI network analysis revealed several hub genes, which might be potential biomarkers for chemotherapy-resistant EOC.

AKT3 was significantly downregulated in chemotherapy-resistant EOC. AKT mediates survival signals that protect cells from apoptosis and is a potentially important therapeutic target. AKT3 levels in various ovarian cancer cell lines correlate with total AKT activity and proliferation rates, suggesting an important role in the genesis of at least one subset of ovarian cancer (Cristiano et al., 2006). Altomare et al. (2004) suggested that low AKT activity is associated with chemotherapy resistance in ovarian cancer. Therefore, AKT3 could be a potential chemopreventive target in ovarian cancer. RTN3 is an integral membrane protein involved in apoptosis. Lee et al. (2009) reported that the overexpression of RTN3 enhances apoptosis mediated by tumor necrosis factorrelated apoptosis-inducing ligand. We speculated that RTN3 was associated with chemotherapy resistance as it was downregulated in chemotherapy-resistant EOC. RAC1 is a GTPase that belongs to the RAS superfamily of small GTP-binding proteins. Wang et al. (2010) implicated Crk/Dock180/ Rac1 in actin cytoskeleton reorganization and thus in cell proliferation, motility, and invasion of the human ovarian cancer cell line SKOV3. EPHA5 is also implicated in the growth, migration, and invasion of cancer cells in culture as well as tumor growth, invasiveness, angiogenesis, and metastasis in vivo (Surawska et al., 2004; Pasquale, 2010). CAV1 is a candidate tumor suppressor gene and a negative regulator of the Ras-p42/44 mitogen-activated kinase cascade (Wiechen et al., 2001). These genes might contribute to resistance to chemotherapy in EOC. Further studies are needed to elucidate the underlying molecular mechanisms of EOC chemotherapy resistance.

Furthermore, we conducted GO enrichment and KEGG pathway enrichment analyses to discover the altered biological functions related to chemotherapy resistance. Cell adhesion, biological adhesion, and intracellular signaling cascade were significantly enriched in the DEGs. There is a relationship between cell adhesion, which is known to protect cells from apoptosis, and drug resistance (Croix and Kerbel, 1997). Sun et al. (2014) reported that gastric cancer cell adhesion to laminin enhances acquired chemotherapeutic drug resistance. Therefore, we speculated that cell adhesion was related to chemotherapy resistance in EOC. The KEGG pathway enrichment analysis indicated that the calcium, MAPK, and B cell receptor signaling pathways were enriched in the DEGs. Pan et al. (2002) found that gamma-synuclein promotes cancer cell survival and inhibits stress- and chemotherapy drug-induced apoptosis by modulating MAPK pathways. In addition, Weir et al. (2007) reported that curcumin induces G2/M phase arrest and apoptosis in cisplatin-resistant human ovarian cancer cells by modulating Akt and p38 MAPK. Furthermore, Coltella et al. (2006) found that p38 MAPK converts hepatocyte growth factor to a death signal that commits ovarian cancer cells to chemotherapy-induced apoptosis. These findings suggest that MAPK signaling is closely related to chemotherapy resistance. The pathway-pathway interaction network could guide future studies to fully uncover the pathways leading to resistance, such as the PI3K/Akt signaling pathway, which is involved in cell cycle progression, apoptosis, and neoplastic transformation (Chang et al., 2003; Carnero et al., 2008). Inhibitors of the PI3K/ Akt signaling pathway could sensitize cancer cells to anti-cancer drugs (Falasca, 2010). Further studies on this pathway might provide novel targets to modulate chemotherapy resistance in EOC. 
Overall, many critical genes associated with chemotherapy resistance in EOC were identified in this study, such as AKT3 and RTN3. Functional enrichment analyses suggested that cell adhesion and MAPK signaling were closely associated with resistance. These findings could advance the understanding of chemotherapy-resistant EOC and provide potential therapeutic targets and biomarkers for clinical applications.

\section{Conflicts of interest}

The authors declare no conflict of interest.

\section{REFERENCES}

Adamcsek B, Palla G, Farkas IJ, Derényi I, et al. (2006). CFinder: locating cliques and overlapping modules in biological networks. Bioinformatics 22: 1021-1023.http://dx.doi.org/10.1093/bioinformatics/btl039

Altomare DA, Wang HQ, Skele KL, De Rienzo A, et al. (2004). AKT and mTOR phosphorylation is frequently detected in ovarian cancer and can be targeted to disrupt ovarian tumor cell growth. Oncogene 23: 5853-5857. http://dx.doi.org/10.1038/sj.onc.1207721

Bachvarov D, L'espérance S, Popa I, Bachvarova M, et al. (2006). Gene expression patterns of chemoresistant and chemosensitive serous epithelial ovarian tumors with possible predictive value in response to initial chemotherapy. Int. J. Oncol. 29: 919-933.

Carnero A, Blanco-Aparicio C, Renner O, Link W, et al. (2008). The PTEN/PI3K/AKT signalling pathway in cancer, therapeutic implications. Curr. Cancer Drug Targets 8: 187-198.http://dx.doi.org/10.2174/156800908784293659

Chang F, Lee JT, Navolanic PM, Steelman LS, et al. (2003). Involvement of PI3K/Akt pathway in cell cycle progression, apoptosis, and neoplastic transformation: a target for cancer chemotherapy. Leukemia 17: 590-603. http://dx.doi.org/10.1038/sj.leu.2402824

Coltella N, Rasola A, Nano E, Bardella C, et al. (2006). p38 MAPK turns hepatocyte growth factor to a death signal that commits ovarian cancer cells to chemotherapy-induced apoptosis. Int. J. Cancer 118: 2981-2990. http://dx.doi.org/10.1002/ijc.21766

Cooke SL and Brenton JD (2011). Evolution of platinum resistance in high-grade serous ovarian cancer. Lancet Oncol. 12: 1169-1174.http://dx.doi.org/10.1016/S1470-2045(11)70123-1

Cristiano BE, Chan JC, Hannan KM, Lundie NA, et al. (2006). A specific role for AKT3 in the genesis of ovarian cancer through modulation of G(2)-M phase transition. Cancer Res. 66: 11718-11725.http://dx.doi.org/10.1158/0008-5472.CAN-06-1968

St Croix B and Kerbel RS (1997). Cell adhesion and drug resistance in cancer. Curr. Opin. Oncol. 9: 549-556. http://dx.doi.org/10.1097/00001622-199711000-00010

Falasca M (2010). PI3K/Akt signalling pathway specific inhibitors: a novel strategy to sensitize cancer cells to anti-cancer drugs. Curr. Pharm. Des. 16: 1410-1416.http://dx.doi.org/10.2174/138161210791033950

Gatti L and Zunino F (2005). Overview of tumor cell chemoresistance mechanisms. Methods Mol. Med. 111: $127-148$.

Helleman J, Smid M, Jansen MP, van der Burg ME, et al. (2010). Pathway analysis of gene lists associated with platinumbased chemotherapy resistance in ovarian cancer: the big picture. Gynecol. Oncol. 117: 170-176. http://dx.doi.org/10.1016/j.ygyno.2010.01.010

Huang DW, Sherman BT, Tan Q, Collins JR, et al. (2007). The DAVID Gene Functional Classification Tool: a novel biological module-centric algorithm to functionally analyze large gene lists. Genome Biol. 8: R183. http://dx.doi.org/10.1186/gb-2007-8-9-r183

Irizarry RA, Hobbs B, Collin F, Beazer-Barclay YD, et al. (2003). Exploration, normalization, and summaries of high density oligonucleotide array probe level data. Biostatistics 4: 249-264.http://dx.doi.org/10.1093/biostatistics/4.2.249

Jazaeri AA, Awtrey CS, Chandramouli GV, Chuang YE, et al. (2005). Gene expression profiles associated with response to chemotherapy in epithelial ovarian cancers. Clin. Cancer Res. 11: 6300-6310. http://dx.doi.org/10.1158/1078-0432.CCR-04-2682

Keshava Prasad TS, Goel R, Kandasamy K, Keerthikumar S, et al. (2009). Human Protein Reference Database--2009 update. Nucleic Acids Res. 37: D767-D772.http://dx.doi.org/10.1093/nar/gkn892

Kohl M, Wiese S and Warscheid B (2011). Cytoscape: software for visualization and analysis of biological networks. Methods Mol. Biol. 696: 291-303.http://dx.doi.org/10.1007/978-1-60761-987-1_18

Konstantinopoulos PA, Spentzos D, Karlan BY, Taniguchi T, et al. (2010). Gene expression profile of BRCAness that correlates 
with responsiveness to chemotherapy and with outcome in patients with epithelial ovarian cancer. J. Clin. Oncol. 28: 3555-3561.http://dx.doi.org/10.1200/JCO.2009.27.5719

Koti M, Gooding RJ, Nuin P, Haslehurst A, et al. (2013). Identification of the IGF1/PI3K/NF $\kappa B / E R K$ gene signalling networks associated with chemotherapy resistance and treatment response in high-grade serous epithelial ovarian cancer. BMC Cancer 13: 549.http://dx.doi.org/10.1186/1471-2407-13-549

L'Espérance S, Popa I, Bachvarova M, Plante M, et al. (2006). Gene expression profiling of paired ovarian tumors obtained prior to and following adjuvant chemotherapy: molecular signatures of chemoresistant tumors. Int. J. Oncol. 29: 5-24.

Lee JT, Lee TJ, Kim CH, Kim NS, et al. (2009). Over-expression of Reticulon 3 (RTN3) enhances TRAIL-mediated apoptosis via up-regulation of death receptor 5 (DR5) and down-regulation of c-FLIP. Cancer Lett. 279: 185-192. http://dx.doi.org/10.1016/j.canlet.2009.01.035

Osterberg L, Levan K, Partheen K, Delle U, et al. (2009). Potential predictive markers of chemotherapy resistance in stage III ovarian serous carcinomas. BMC Cancer 9: 368.http://dx.doi.org/10.1186/1471-2407-9-368

Pan ZZ, Bruening W, Giasson BI, Lee VM, et al. (2002). Gamma-synuclein promotes cancer cell survival and inhibits stressand chemotherapy drug-induced apoptosis by modulating MAPK pathways. J. Biol. Chem. 277: 35050-35060. http://dx.doi.org/10.1074/jbc.M201650200

Pasquale EB (2010). Eph receptors and ephrins in cancer: bidirectional signalling and beyond. Nat. Rev. Cancer 10: 165-180. http://dx.doi.org/10.1038/nrc2806

<unknown>Smyth GK (2004). Linear models and empirical bayes methods for assessing differential expression in microarray experiments. Stat. Appl. Genet. Mol. Biol. 3: Article3.</unknown>

<bok>Stewart BW and Wild CP (2014). World Cancer Report 2014. International Agency for Research on Cancer.</bok>

Sun L, Liu L, Liu X, Wang Y, et al. (2014). Gastric cancer cell adhesion to laminin enhances acquired chemotherapeutic drug resistance mediated by MGr1-Ag/37LRP. Oncol. Rep. 32: 105-114.

Surawska H, Ma PC and Salgia R (2004). The role of ephrins and Eph receptors in cancer. Cytokine Growth Factor Rev. 15: 419-433. http://dx.doi.org/10.1016/j.cytogfr.2004.09.002

Wang H, Linghu H, Wang J, Che YL, et al. (2010). The role of Crk/Dock180/Rac1 pathway in the malignant behavior of human ovarian cancer cell SKOV3. Tumour Biol. 31: 59-67.http://dx.doi.org/10.1007/s13277-009-0009-9

Weir NM, Selvendiran K, Kutala VK, Tong L, et al. (2007). Curcumin induces G2/M arrest and apoptosis in cisplatin-resistant human ovarian cancer cells by modulating Akt and p38 MAPK. Cancer Biol. Ther. 6: 178-184. http://dx.doi.org/10.4161/cbt.6.2.3577

Wiechen K, Diatchenko L, Agoulnik A, Scharff KM, et al. (2001). Caveolin-1 is down-regulated in human ovarian carcinoma and acts as a candidate tumor suppressor gene. Am. J. Pathol. 159: 1635-1643.

http://dx.doi.org/10.1016/S0002-9440(10)63010-6 